\title{
Estilos de aprendizaje de estudiantes de medicina en universidades latinoamericanas y españolas: relación con los contextos geográficos y curriculares
}

\author{
G. Díaz-Véliza, S. Mora a , J.V. Lafuente-Sáncheze, P.A. Gargiulob, \\ R. Bianchi ${ }^{b}$, C. Terán ${ }^{c}$, D. Gorena c, J. Arce ${ }^{d}$, J.F. Escanero-Marcen ${ }^{\dagger}$
}

Objetivo. Determinar si los estilos de aprendizaje (EA) de los estudiantes de medicina se correlacionan con el contexto geográfico, con el contexto curricular o con el nivel de la carrera. Sujetos y métodos. El estudio se realizó en 490 estudiantes de las Escuelas de Medicina de las Universidades de Chile (Santiago, Chile), Nacional de Cuyo (Mendoza, Argentina), San Francisco Xavier (Sucre, Bolivia), Zaragoza y País Vasco (España). Se aplicó el cuestionario Honey-Alonso, que valora la preferencia por cada uno de cuatro EA: activo, reflexivo, teórico y pragmático. También se evaluó el EA de acuerdo al modelo de Kolb. Resultados. Al relacionar el EA con el contexto geográfico se observó que mientras los estudiantes de universidades españolas muestran un estilo preferentemente asimilador, siguiendo la denominación de Kolb, para Chile fue el acomodador y para Bolivia los estudiantes se distribuyen entre los estilos asimilador y divergente. Al comparar la distribución de los EA durante el tercer curso de medicina en dos facultades que poseen diferente currículo, no se observaron diferencias significativas. Los EA en una Facultad de Medicina con un currículo basado en asignaturas (Chile) no mostraron diferencias en los tres cursos del estudio $\left(1 .^{\circ}, 3^{\circ}\right.$ y $\left.5 .{ }^{\circ}\right)$, siendo preferentes los estilos reflexivo y teórico. Conclusiones. El estudio permitió establecer diferencias significativas entre los estilos de aprendizaje de los estudiantes de Medicina en relación con el contexto geográfico, más que con los diferentes currículos, o a lo largo de los distintos cursos de la carrera.

Palabras clave. CHAEA. Contexto curricular. Contexto geográfico. Estilos de aprendizaje. Estudiantes de medicina. Kolb.

\section{Medical students learning styles in Latin American and Spanish universities: relation with geographical and curricular contexts}

Aim. To establish a correlation between medical student learn- ing styles (LS) and the geographical context, the curricular context and different academic levels. Subjects and methods. The study was performed in 490 undergraduate students from Medical Schools of the Universities of Chile (Santiago, Chile), Nacional de Cuyo (Mendoza, Argentina), San Francisco Xavier (Sucre, Bolivia), Zaragoza and País Vasco (Spain). The instrument used was the Honey-Alonso learning style questionnaire that assesses the student preference for one of four LS: active, reflexive, theoretic and pragmatic. In addition, LS according to the Kolb inventory were also assessed. Results. Using the Kolb inventory, significant differences were found when the LS were correlated with the geographical context. While Spanish students showed a high preference for the assimilator style of learning, Chilean students resulted to be mainly accommodators, and Bolivian students were both assimilators and divergent. Comparing the LS distribution during the third course in two universities with different curricula (problem and lecture based learning), there were no significant differences. LS of medical students from a Medical School with a lecture based curriculum (University of Chile) were not significantly different during the first, the third and the fifth level of their undergraduate students. They showed a significant preference for reflexive and theoretic styles of learning. Conclusions. The present study allowed demonstrating that significant differences among the styles of learning of medical students correlated with the geographical context more than with the different curricula, or along the different courses of the career.

Key words. CHAEA. Curricular context. Geographical context. Kolb. Learning styles. Undergraduate medical students.

\section{Introducción}

Mucho se ha hablado y escrito acerca de la necesidad de desarrollar en los estudiantes la competencia de aprendizaje autónomo y reflexivo [1]

\footnotetext{
a Facultad de Medicina. Universidad de Chile. Santiago, Chile.

b Facultad de Ciencias Médicas. Universidad Nacional de Cuyo. Mendoza, Argentina. cDepartamento Salud Pública. Universidad Andina Simón Bolívar Sucre, Bolivia.

dFacultad de Medicina. Universidad San Francisco Xavier. Sucre, Bolivia.

e Facultad de Medicina y Odontología. Universidad del País Vasco. Bilbao, Vizcaya, España. ${ }^{f}$ Facultad de Medicina. Universidad de Zaragoza. Zaragoza, España.
}

Correspondencia Prof. Gabriela Díaz-Véliz. Programa de Farmacología Molecular y Clínica. Instituto de Ciencias Biomédicas. Facultad de Medicina. Universidad de Chile. P.O. Box 16038 . Santiago-9, Chile.

E-mail gdiaz@med.uchile.cl

Agradecimientos A la Agencia Española de Cooperación Internacional y Desarrollo (AECID) y a las autoridades académicas de las Facultades de Medicina de las universidades implicadas en el estudio, sin olvidar la desinteresada participación de nuestros estudiantes. Trabajo financiado con el proyecto B012377/07 de AECID (Ministerio de Asuntos Exteriores de España). 
y de cómo debe evolucionar el rol del profesor para dejar de ser una mera figura transmisora de información y pasar a ser un promotor del aprendizaje [2]. En este enfoque, es cada vez más importante la comprensión, tanto por parte del estudiante como del profesor, de qué es el aprendizaje, cómo se produce, de qué depende y en qué diferentes formas puede darse en un individuo [2]. El conocimiento y comprensión por parte del estudiante de su propio estilo de aprendizaje le permitirá tomar conciencia de las estrategias cognitivas que emplea y de cuales son las más adecuadas en cada caso; además, se trata de uno de los factores claves para desarrollar su capacidad de aprender a aprender [3]. Por otra parte, el conocimiento y comprensión de los estilos de aprendizaje de los estudiantes, por parte del profesor, facilitará la toma de decisiones académicas fundamentales y la orientación al estudiante para identificar posibles deficiencias y dificultades, permitiéndole mejorar su rendimiento [1]. En esta línea, el cómo los estudiantes aprenden es de singular importancia, ya que una determinada estrategia de enseñanza aplicada por un docente no producirá el mismo efecto de aprendizaje en todos los individuos [4].

Entre las clasificaciones de estilos de aprendizaje, una de las más utilizadas ha sido el Learning Style Inventory de Kolb (LSI), basado en la teoría del aprendizaje experiencial $[5,6]$. Esta teoría considera que el proceso de aprender incluye un recorrido cíclico por cuatro etapas sucesivas: tener una experiencia, reflexionar sobre ella, extraer conclusiones y planificar los pasos para su aplicación. En la práctica, la mayoría de nosotros tendemos a especializarnos en una, o como mucho dos, de esas cuatro fases, por lo que se pueden diferenciar cuatro tipos de estudiantes, dependiendo de la fase en la que prefieran trabajar. A partir del ciclo de aprendizaje Kolb, en su inventario, clasifica a los aprendedores en cuatro categorías, según sus particulares estilos de aprendizaje:

- Divergentes: los que se basan en experiencias concretas y observación reflexiva.

- Convergentes: los que utilizan la conceptualización abstracta y la experimentación activa.

- Asimiladores: los que usan la conceptualización abstracta y la observación reflexiva.

- Acomodadores: los se basan en la experiencia concreta y la experimentación activa.
Basándose en el LSI de Kolb, Honey y Munford desarrollaron el Learning Style Questionnaire (LSQ) [7], el que posteriormente fue adaptado al contexto académico español por Alonso, Gallego y Honey con el nombre de CHAEA [3]. Estos autores realizaron un análisis con un mayor número de variables y concluyeron que los estilos de aprendizaje podrían clasificarse en cuatro tipos: activo, reflexivo, teórico y pragmático, que se podrían asimilar a las fases del ciclo de aprendizaje de Kolb:

- Estilo activo: personas que se caracterizan por buscar nuevas experiencias. Son de mente abierta, muy activos, comprometidos con el trabajo del grupo y se involucran con entusiasmo en nuevas tareas.

- Estilo reflexivo: personas observadoras y que consideran las experiencias desde diferentes ángulos. Analizan con cuidado antes de llegar a una conclusión y su filosofía se basa en ser prudentes.

- Estilo teórico: personas que buscan la racionalidad y la objetividad, huyendo de todo lo que es ambiguo y subjetivo. Predomina el pensamiento lógico, el ser perfeccionista y la integración de los conceptos en teorías lógicas.

- Estilo pragmático: personas en las que predomina la aplicación práctica de las ideas. Les gusta actuar con seguridad y experimentar el aspecto positivo de las nuevas ideas.

El presente estudio tiene el propósito de identificar los estilos de aprendizaje de estudiantes de medicina situados en diferentes contextos geográficos (España frente a Latinoamérica). Con este objetivo, se aplicó el cuestionario de Honey-Alonso, y su posterior conversión al modelo de Kolb, para comparar los estilos de aprendizaje de estudiantes de medicina de universidades de España (Universidades del País Vasco y Zaragoza), Argentina (Universidad Nacional de Cuyo, Mendoza), Bolivia (Universidad de San Francisco Xavier, Sucre) y Chile (Universidad de Chile).

Como objetivos secundarios se estudió la influencia de diferentes modelos curriculares (currículo basado en problemas frente a currículo basado en asignaturas) sobre los estilos de aprendizaje y la correlación entre éstos y el nivel de la carrera en que se encuentran los estudiantes (cursos primero, tercero y quinto). 


\section{Sujetos y métodos}

\section{Descripción de la muestra}

Este estudio se realizó en 490 estudiantes de las Escuelas/Facultades de Medicina de las Universidades de Chile (Santiago, Chile), Nacional de Cuyo (Mendoza, Argentina), San Francisco Xavier (Sucre, Bolivia) y Zaragoza y País Vasco (España), los cuales fueron distribuidos, de acuerdo con los objetivos diseñados, de la siguiente manera:

Para determinar la influencia del contexto geográfico, se correlacionaron los estilos de aprendizaje de los estudiantes del primer curso de Santiago, Chile $(n=61)$, Bilbao, España $(n=$ $87)$, Zaragoza, España $(n=77)$ y Sucre, Bolivia $(n=61)$, todas ellas con currículos tradicionales.

Para la correlación entre los estilos de aprendizaje y el contexto curricular, se compararon los estilos de aprendizaje de los estudiantes de tercer curso de una Facultad de Medicina con un currículo tradicional basado en asignaturas (Chile, $n=68)$ con otro currículo basado en problemas (Argentina, $n=70$ ).
Para el estudio de los estilos de aprendizaje de los estudiantes con un currículo tradicional, basado en asignaturas, en diferentes niveles académicos, se utilizaron los estudiantes de primer $(n=61)$, tercer $(n=68)$ y quinto curso $(n=66)$ de la Facultad de Medicina de la Universidad de Chile.

\section{Instrumento utilizado}

Los estilos de aprendizaje se determinaron aplicando el Cuestionario Honey Alonso de Estilos de Aprendizaje (CHAEA) [3], que valora la preferencia por cada uno de los siguientes cuatro estilos de aprendizaje: activo, reflexivo, teórico y pragmático. El cuestionario consta de 80 ítems, breves y dicotómicos (Tabla I) de los cuales 20 corresponden a cada estilo de aprendizaje y están distribuidos aleatoriamente. La puntuación absoluta que se obtiene en cada grupo de 20 ítems indica el nivel que alcanza el individuo en cada uno de los cuatro estilos. La representación sobre un eje cartesiano permite determinar cada estilo preferente.

Tabla I. Ítems del cuestionario Honey-Alonso de estilos de aprendizaje [3].

1. Tengo fama de decir lo que pienso claramente y sin rodeos

2. Estoy seguro de lo que es bueno y lo que es malo, lo que esta bien y lo que esta mal

3. Muchas veces actúo sin mirar las consecuencias

4. Normalmente trato de resolver los problemas metódicamente y paso a paso

5. Creo que los formalismos coartan y limitan la actuación libre de las personas

6. Me interesa saber cuales son los sistemas de valores de los demás y con qué criterios actúan

7. Pienso que el actuar intuitivamente puede ser siempre tan valido como actuar reflexivamente

8. Creo que lo más importante es que las cosas funcionen

9. Procuro estar al tanto de lo que ocurre aquí y ahora

10. Disfruto cuando tengo tiempo para preparar mi trabajo y realizarlo a conciencia

11. Estoy a gusto siguiendo un orden, en las comidas, en el estudio, haciendo ejercicio regularmente

12. Cuando escucho una nueva idea enseguida comienzo a pensar como ponerla en práctica

13. Prefiero las ideas originales y novedosas aunque no sean prácticas

14. Admito y me ajusto a las normas solo si me sirven para lograr mis objetivos

15. Normalmente encajo bien con personas reflexivas, y me cuesta sintonizar con personas demasiado espontáneas, imprevisibles

16. Escucho con más frecuencia que hablo

17. Prefiero las cosas estructuradas a las desordenadas

18. Cuando poseo cualquier información, trato de interpretarla bien antes de manifestar alguna conclusión

19. Antes de hacer algo estudio con cuidado sus ventajas e inconvenientes

20. Crezco con el reto de hacer algo nuevo y diferente

21. Casi siempre procuro ser coherente con mis criterios y sistemas de valores. Tengo principios y los sigo

22. Cuando hay una discusión no me gusta ir con rodeos

23. Me disgusta implicarme afectivamente en mi ambiente de trabajo. Prefiero mantener relaciones distantes

24. Me gustan más las personas realistas y concretas que las teóricas 


\section{Tabla I. Ítems del cuestionario Honey-Alonso de estilos de aprendizaje [3] (cont.).}

25. Me gusta ser creativo, romper estructuras

26. Me siento a gusto con personas espontáneas y divertidas

27. La mayoría de las veces expreso abiertamente cómo me siento

28. Me gusta analizar y dar vueltas a las cosas

29. Me molesta que la gente no se tome en serio las cosas

30. Me atrae experimentar y practicar las últimas técnicas y novedades

31. Soy cauteloso a la hora de sacar conclusiones

32. Prefiero contar con el mayor número de fuentes de información. Cuantos más datos reúna para reflexionar, mejor

33. Tiendo a ser perfeccionista

34. Prefiero oír las opiniones de los demás antes de exponer la mía

35. Me gusta afrontar la vida espontáneamente y no tener que planificar todo previamente

36. En las discusiones me gusta observar cómo actúan los demás participantes

37. Me siento incómodo con las personas calladas y demasiado analíticas

38. Juzgo con frecuencia las ideas de los demás por su valor práctico

39. Me agobio si me obligan a acelerar mucho el trabajo para cumplir un plazo.

40. En las reuniones apoyo las ideas prácticas y realistas

41. Es mejor gozar del momento presente que deleitarse pensando en el pasado o en el futuro

42. Me molestan las personas que siempre desean apresurar las cosas

43. Aporto ideas nuevas y espontáneas en los grupos de discusión

44. Pienso que son más conscientes las decisiones fundamentadas en un minucioso análisis que las basadas en la intuición

45. Detecto frecuentemente la inconsistencia y puntos débiles en las argumentaciones de los demás

46. Creo que es preciso saltarse las normas muchas más veces que cumplirlas

47. A menudo caigo en cuenta de otras formas mejores y más prácticas de hacer las cosas

48. En conjunto hablo más que escucho

49. Prefiero distanciarme de los hechos y observarlos desde otras perspectivas

50. Estoy convencido que deber imponerse la lógica y el razonamiento

51. Me gusta buscar nuevas experiencias

52. Me gusta experimentar y aplicar las cosas

53. Pienso que debemos llegar pronto al grano, al meollo de los temas

54. Siempre trato de conseguir conclusiones e ideas claras

55. Prefiero discutir cuestiones concretas y no perder el tiempo con charlas vacías

56. Me impaciento cuando me dan explicaciones irrelevantes e incoherentes

57. Compruebo antes si las cosas funcionan realmente

58. Hago varios borradores antes de la redacción definitiva de un trabajo

59. Soy consciente de que en las discusiones ayudo a mantener a los demás centrados en el tema, evitando divagaciones

60. Observo que, con frecuencia, soy uno de los más objetivos y desapasionados en las discusiones

61. Cuando algo va mal le quito importancia y trato de hacerlo mejor

62. Rechazo ideas originales y espontáneas si no las veo prácticas

63. Me gusta sopesar diversas alternativas antes de tomar una decisión

64. Con frecuencia miro hacia delante para prever el futuro

65. En los debates y discusiones prefiero desempeñar un papel secundario antes que ser el/la líder o el/la que más participa

66. Me molestan las personas que no actúan con lógica

67. Me resulta incomodo tener que planificar y prever las cosas

68. Creo que el fin justifica los medios en muchos casos

69. Suelo reflexionar sobre los asuntos y problemas

70. El trabajar a conciencia me llena de satisfacción y orgullo

71. Ante los acontecimientos trato de descubrir los principios y teorías en que se basan

72. Con tal de conseguir el objetivo que pretendo soy capaz de herir sentimientos ajenos

73. No me importa hacer todo lo necesario para que sea efectivo mi trabajo

74. Con frecuencia soy una de las personas que más anima las fiestas

75. Me aburro enseguida con el trabajo metódico y minucioso

76. La gente con frecuencia cree que soy poco sensible a sus sentimientos

77. Suelo dejarme llevar por mis intuiciones

78. Si trabajo en grupo procuro que se siga un método y un orden

79. Con frecuencia me interesa averiguar lo que piensa la gente

80. Esquivo los temas subjetivos, ambiguos y poco claros 
Tabla II. Equivalencia de estilos de aprendizaje entre ambos modelos.

\begin{tabular}{cc}
\hline LSQ de Honey-Alonso & LSI de Kolb \\
\hline Activo & Experiencia concreta \\
\hline Reflexivo & Observación reflexiva \\
\hline Teórico & Conceptualización abstracta \\
\hline Pragmático & Experimentación activa \\
\hline
\end{tabular}

\section{Conversión al modelo de Kolb}

Los estilos de Alonso, Gallego y Honey [7] pueden convertirse en los estilos de aprendizaje de Kolb [5,6]. La teoría del aprendizaje experiencial del mencionado autor [8] señala que el aprendizaje es el proceso por el cual se crea conocimiento a través de la transformación de la información o experiencia. Así, el conocimiento resultaría de la combinación de obtención y transformación de la información. De esta forma, la teoría establece dos modos dialécticamente relacionados para obtener información: la experiencia concreta y la conceptualización abstracta, y dos modos dialécticamente relacionados para transformar la información: la observación reflexiva y la experimentación activa. El proceso es idealizado como un ciclo o espiral de aprendizaje, donde el aprendedor pasa por las fases de experimentación, reflexión, pensamiento y acción, que responde a la situación de aprendizaje y a lo que está siendo aprendido [9]. Estas cuatro fases se corresponderían con los cuatro estilos de Honey-Alonso.

En la tabla II se indica la equivalencia de estilos de aprendizaje entre ambos modelos.

Para evidenciar las diferencias debidas a distintos contextos geográficos y curriculares en cuanto a la combinación de obtención y transformación de la información, se realizó un gráfico de dispersión con la ubicación de cada estudiante dentro de su estilo de aprendizaje preponderante de acuerdo al modelo de Kolb. De esta manera, los valores de $x$ e $y$ necesarios para su representación se obtuvieron con la diferencia neta entre las puntuaciones del cuestionario Honey-Alonso para cada par de estilos antagónicos (teórico frente a activo, y pragmático frente a reflexivo). La cuenta final del número de estudiantes adjudicados a cada cuadrante determinó el porcentaje para cada estilo.

\section{Tratamiento estadístico}

Para procesar la información de los cuestionarios se elaboró una base de datos en el programa Excel y se realizó un análisis estadístico con el programa GraphPad Prism v.5. Dicho análisis consistió en la comparación de las puntuaciones medias obtenidas en los cuatro estilos de aprendizaje, según Honey-Alonso, en los diferentes cursos de las universidades involucradas en el estudio. Posteriormente, se transformaron los datos para obtener las puntuaciones de acuerdo al modelo de Kolb como fue señalado anteriormente. Se aplicó el análisis de varianza de una vía (ANOVA) para cada estilo, seguido por la prueba de Bonferroni para comparaciones múltiples, teniendo en cuenta la homogeneidad de las varianzas. La comparación de los porcentajes se realizó con la prueba de chi al cuadrado. En cada caso se consideró como significativo una $p \leq 0,05$.

\section{Resultados}

Relación entre estilo de aprendizaje y contexto geográfico

Para analizar la influencia del diferente contexto geográfico (España y Latinoamérica) se eligie- 


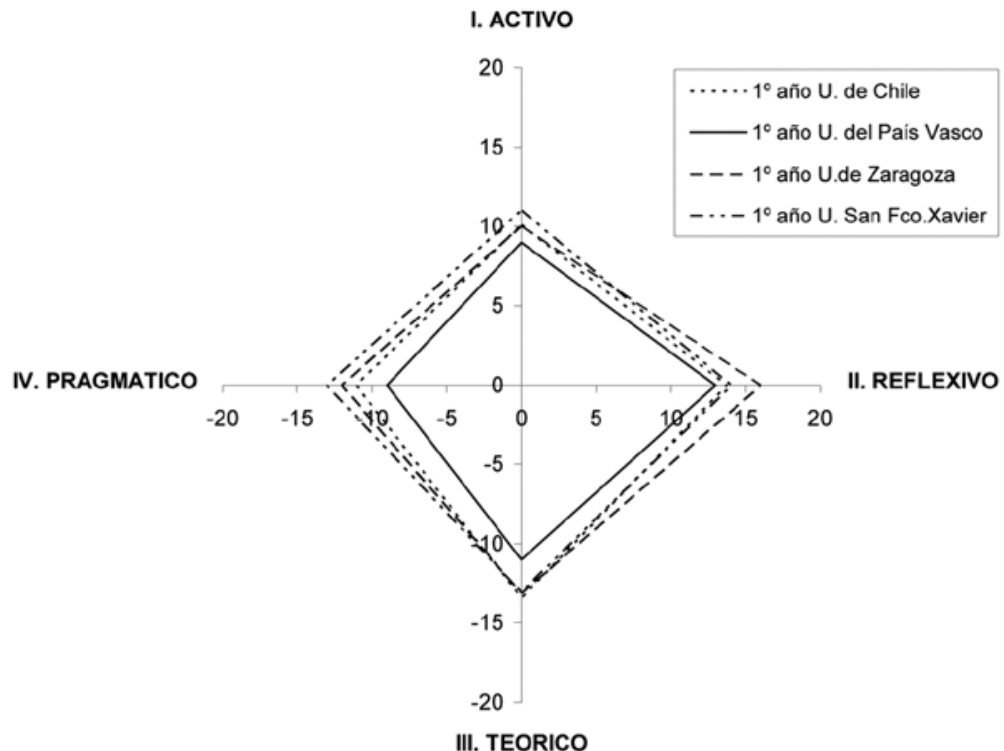

\begin{tabular}{|c|c|c|c|c|}
\hline Universidad & Activo & Reflexivo & Teórico & Pragmático \\
\hline U. de Chile & $10,1 \pm 0,4$ & $13,7 \pm 0,4 *$ & $13,4 \pm 0,3 *$ & $11,1 \pm 0,4$ \\
\hline $\begin{array}{c}\text { U. del País } \\
\text { Vasco }\end{array}$ & $8,7 \pm 0,3$ & $13,0 \pm 0,3 *$ & $11,2 \pm 0,3 *$ & $9,1 \pm 0,3$ \\
\hline $\begin{array}{c}\text { U. de } \\
\text { Zaragoza }\end{array}$ & $10,3 \pm 0,4$ & $15,6 \pm 0,3 *$ & $13,3 \pm 0,3 *$ & $11,7 \pm 0,4 *$ \\
\hline $\begin{array}{c}\text { U. San Fco. } \\
\text { Xavier }\end{array}$ & $10,9 \pm 0,4$ & $13,9 \pm 0,4 *$ & $12,7 \pm 0,4 *$ & $13,2 \pm 0,3 *$ \\
\hline
\end{tabular}

Figura 1. Estilos de aprendizaje y contexto geográfico: puntuaciones promedio obtenidas para cada estilo de aprendizaje en estudiantes de primer año de la Facultad de Medicina de la Universidad de Chile (Chile), Universidad del País Vasco (España), Universidad de Zaragoza (España) y Universidad San Francisco Xavier (Bolivia). ${ }^{*} p<0,05$ comparando entre los estilo de aprendizaje de cada universidad.

ron universidades que poseen el mismo sistema curricular. En la figura 1 se muestran los resultados sobre un eje cartesiano donde se aprecia que los estudiantes del primer curso de la Universidad de Chile (Chile) y del País Vasco (España) presentan un significativo predominio de los estilos reflexivo y teórico sobre los estilos activo y pragmático. En las Universidades de Zaragoza (España) y San Francisco Xavier de Sucre (Bolivia), los estilos reflexivo, teórico y pragmático fueron significativamente superiores al estilo activo. Estas diferencias significativas dentro de las universidades del estudio nos orientaron a analizar mas en detalle las diferencias existentes entre ellas.
En la figura 2, al comparar los estilos de aprendizaje preferentes de los estudiantes de diferentes contextos geográficos, se evidencian diferencias significativas entre todos ellos, ANOVA $F_{(3,282)}=$ 8,01 para el estilo activo, 9,98 para el reflexivo, 11,41 para el teórico y 27,73 para el pragmático, en todos los casos $p<0,0001$. Los estudiantes de Medicina de la Universidad del País Vasco muestran una puntuación promedio significativamente menor al de los alumnos de las otras universidades del estudio en lo que a los estilos activo, teórico y pragmático se refiere. Los de la Universidad de Zaragoza tienen una puntuación promedio para el estilo reflexivo significativamente superior al resto, en tanto que los de la Universidad San 


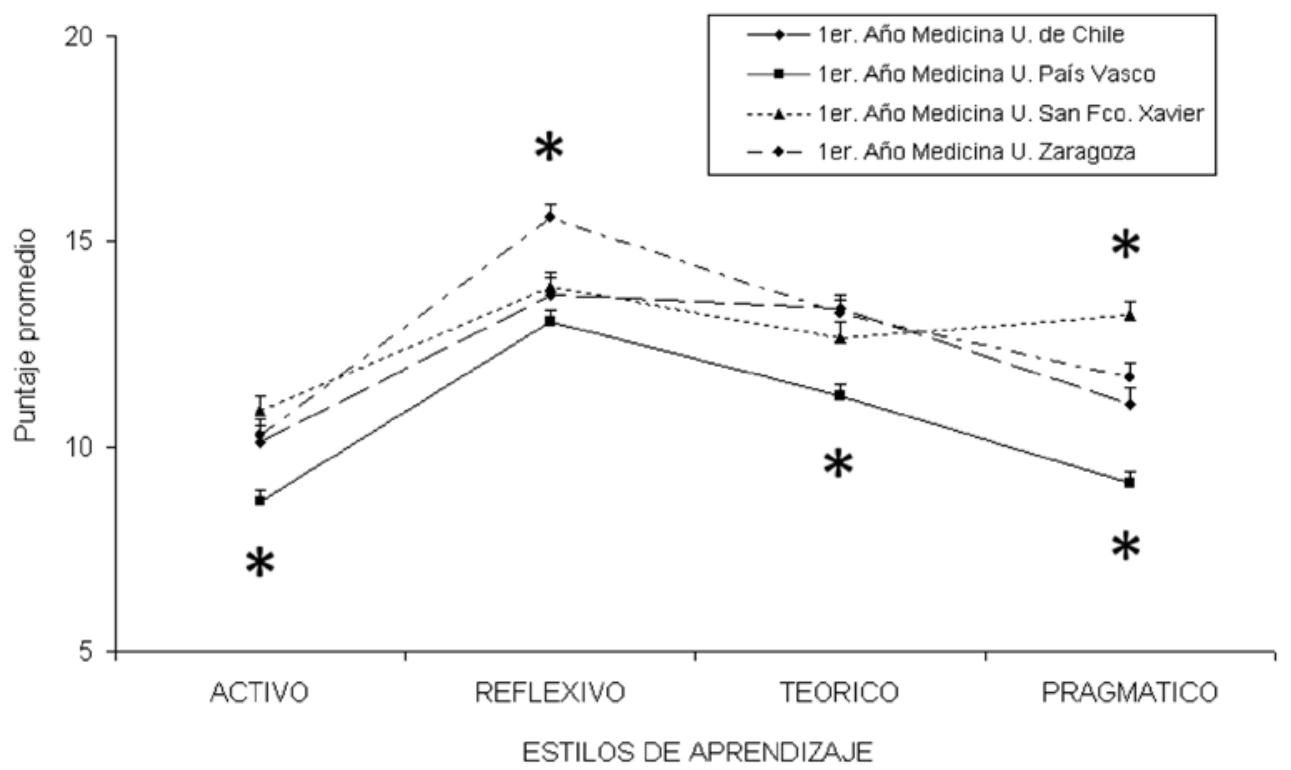

Figura 2. Estilos de aprendizaje y contexto geográfico: puntuaciones promedio obtenidas para cada estilo de aprendizaje en estudiantes de primer año de la Facultad de Medicina de la Universidad de Chile (Chile), Universidad del País Vasco (España), Universidad de Zaragoza (España) y Universidad San Francisco Xavier (Bolivia). ${ }^{*} p<0,05$ comparando las diferentes universidades dentro de cada estilo de aprendizaje.

Francisco Xavier muestran una puntuación significativamente superior a las otras universidades en el estilo pragmático.

Al combinar las preferencias en la adquisición y procesamiento de la información y confeccionar el gráfico de dispersión con la ubicación de cada estudiante dentro de su estilo de aprendizaje preponderante, se observa que las cuatro universidades muestran una distribución significativamente diferente en los estilos de aprendizaje (Fig. 3). Los estudiantes de la Universidad de Chile se concentran en el estilo acomodador (66\%), en cambio en las universidades españolas los estudiantes se concentran significativamente en el estilo asimilador (65 y 62\%), estilo que también es el preferente en los estudiantes bolivianos (39\%), aunque en éstos últimos el segundo estilo significativamente preferente es el divergente (30\%).

\section{Relación entre estilo de aprendizaje y contexto curricular}

Al comparar la distribución de los estilos de aprendizaje preferentes de los estudiantes durante el tercer curso de medicina en dos Facultades que poseen diferente currículo: basado en asig- naturas (Universidad de Chile, Chile) y basado en problemas (Universidad Nacional de Cuyo, Argentina), se observa un significativo predominio de los estilos reflexivo y teórico en ambas Facultades (Fig. 4). Se aprecia que no hay diferencias significativas entre ambas universidades, por lo que el diferente currículo no parece tener una influencia significativa en los estilos de aprendizaje de los estudiantes.

Con el objeto de poner de manifiesto si existía alguna diferencia en la combinación de las preferencias en la adquisición y procesamiento de la información se confeccionó la figura 5 que muestra el gráfico de dispersión con la ubicación de cada estudiante dentro de su estilo de aprendizaje preponderante. Como se mencionó anteriormente, los valores de $x$ e $y$ necesarios para su representación se obtuvieron con la diferencia neta entre las puntuaciones del cuestionario Honey-Alonso para cada par de estilos antagónicos (teórico frente a activo, y pragmático frente a reflexivo). La cuenta final del número de adjudicados a cada cuadrante determinó el porcentaje para cada estilo. Un significativo número de estudiantes, de ambas universidades, se concentra en el estilo acomodador lo cual significa que el diferente cu- 


\section{EC / Activo}

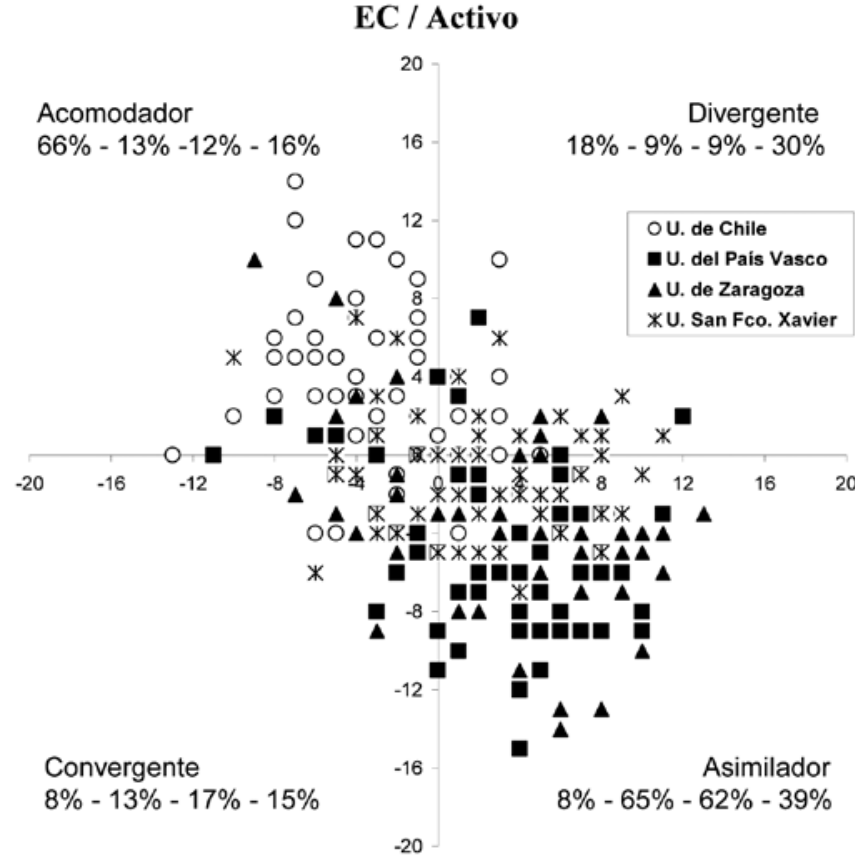

OR / Reflexivo

EA / Pragmático

CA / Teórico

Figura 3. Estilos de aprendizaje y contexto geográfico: diagrama de dispersión con el estilo preponderante de cada estudiante de primer curso de la Facultad de Medicina de la Universidad de Chile (Chile), Universidad del País Vasco (España), Universidad de Zaragoza (España) y Universidad San Francisco Xavier (Bolivia). Los ejes indican estilos según Kolb/Honey-Alonso. Los porcentajes señalan la proporción de individuos en cada cuadrante (perfil de preferencias individuales).

rriculum no modifica significativamente la forma en que los estudiantes adquieren y procesan la información recibida en la facultad.

\section{Estilos de aprendizaje en una Facultad de Medicina con un currículo basado en asignaturas (Universidad de Chile)}

En la figura 6 se muestran los resultados de los diferentes cursos académicos de la Universidad de Chile sobre un eje cartesiano. En el perfil de aprendizaje de los estudiantes, en los tres cursos, se observa una significativa mayor puntuación en los estilos reflexivo y teórico versus los estilos pragmático y activo. $\mathrm{Al}$ analizar cada estilo preferente de aprendizaje, no se encuentran diferencias significativas entre los 3 cursos: ANOVA $F_{(2,192)}=0,85$ para el estilo activo, 0,10 para el reflexivo, 0,36 para el teórico y 0,12 para el pragmático.

\section{Discusión}

En la última década se han realizado importantes esfuerzos por conceptualizar las características de los estilos de aprendizaje de los estudiantes universitarios de Ciencias de la Salud [1,4,10-13]. Los resultados obtenidos en esta investigación indican que los estudiantes de Medicina tienen preferencia por los estilos reflexivo y teórico, los que muestran bastante estabilidad a lo largo de su formación académica y que son, aparentemente, independientes de los contextos geográficos y curriculares en que se encuentran inmersos. Otras investigaciones realizadas en estudiantes de Medicina de la Universidad de Zaragoza [11] y estudios transversales llevados a cabo en estudiantes de Ciencias de la Salud de la Universidad de Cádiz [12] y de la Escuela Universitaria de Enfermería La Paz de Madrid [13] también han mostrado una tendencia hacia un estilo de aprendizaje reflexivo y teórico. 


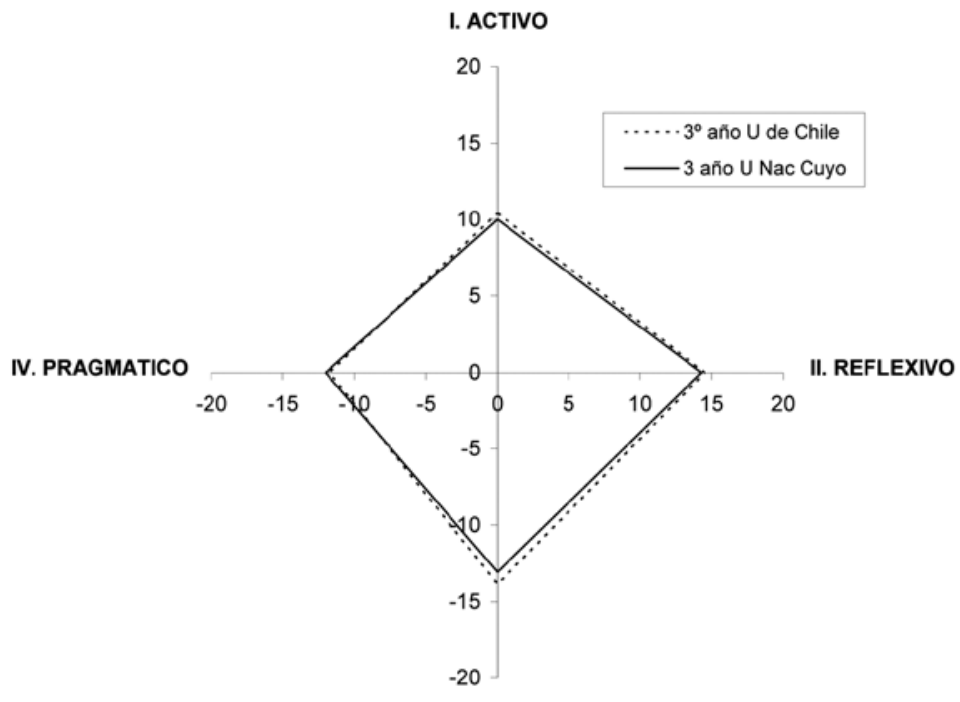

III. TEORICO

\begin{tabular}{|c|c|c|c|c|}
\hline Universidad & Activo & Reflexivo & Teórico & Pragmático \\
\hline U. de Chile & $10,4 \pm 0,5$ & $14,5 \pm 0,4 *$ & $13,9 \pm 0,4 *$ & $11,7 \pm 0,4$ \\
\hline U. Nac. de Cuyo & $10,2 \pm 0,4$ & $14,4 \pm 0,4 *$ & $13,5 \pm 0,3 *$ & $11,5 \pm 0,3$ \\
\hline
\end{tabular}

Figura 4. Estilos de aprendizaje y contexto curricular: puntuaciones promedio obtenidas para cada estilo de aprendizaje en la muestra de estudiantes de tercer año de la Facultad de Medicina de la Universidad de Chile (Chile) y de la Universidad Nacional de Cuyo (Argentina). ${ }^{*} p<0,05$ comparando entre los estilo de aprendizaje de cada universidad. No se aprecian diferencias significativas entre las dos universidades para cada estilo de aprendizaje.

Se podría especular que la mayoría de los estudiantes de Ciencias de la Salud posee un perfil de aprendizaje que los diferencia, por ejemplo, de los estudiantes de Bellas Artes o Filosofía [3]. Es muy probable que este perfil se haya forjado antes del ingreso a la Universidad, posiblemente durante la enseñanza secundaria, el cual no cambia a medida que los estudiantes avanzan en su formación, de acuerdo con los resultados obtenidos en los tres cursos de la Universidad de Chile. Más aún, es probable que las estrategias didácticas utilizadas por los docentes, favorezcan el aprendizaje y rendimiento de los estudiantes con estilos reflexivos y teóricos, potenciando así las preferencias de los estudiantes por dichos estilos.

Es interesante que al comparar el tercer curso de universidades con diferentes currículos, uno tradicional basado en asignaturas (Universidad de Chile) y otro basado en problemas (Universidad Nacional de Cuyo), los resultados muestran que los estudiantes de ambas universidades siguen manteniendo una preferencia similar por los estilos reflexivo y teórico. Se podría pensar que los estudiantes que ingresan a la carrera de medicina con una preferencia preadquirida por el estilo reflexivo-teórico no lo modifican, a pesar de que el estilo de enseñanza sea muy diferente y supuestamente uno de ellos orientado al desarrollo homogéneo de todas las capacidades de aprendizaje. En este mismo contexto, la conversión a los estilos de aprendizaje de Kolb, permitió establecer que los estudiantes de ambas universidades corresponden mayoritariamente el estilo acomodador, es decir, que en su aprendizaje combinan el pensamiento concreto con el procesamiento activo. 


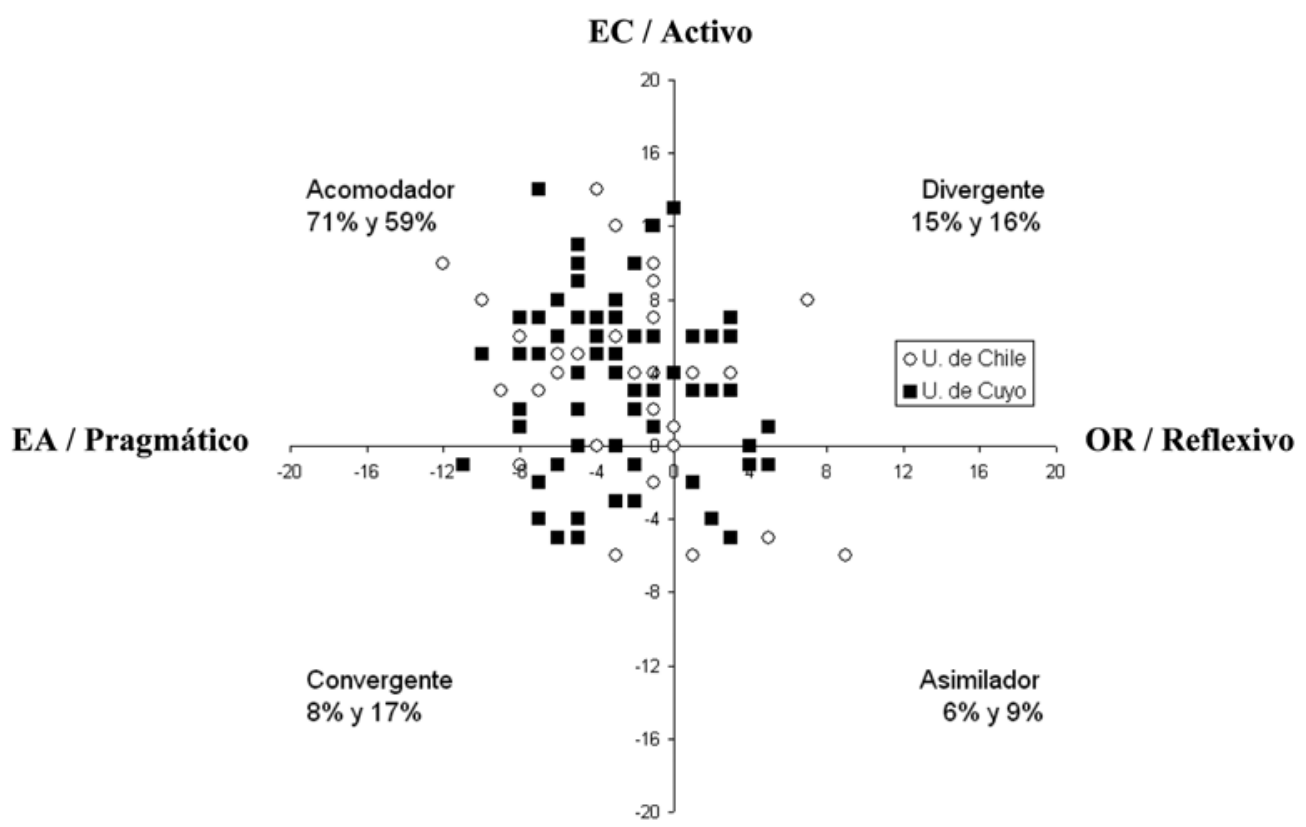

CA / Teórico

Figura 5. Estilos de aprendizaje y contexto curricular: diagrama de dispersión con el estilo preponderante de cada estudiante de tercer curso de las Facultades de Medicina de la Universidad de Chile (Chile) y Universidad Nacional de Cuyo (Argentina). Los ejes indican estilos según Kolb/Honey-Alonso. Los porcentajes señalan la proporción de individuos en cada cuadrante (perfil de preferencias individuales).

El tener preferencia por uno o dos estilos de aprendizaje no es malo ni bueno intrínsecamente. Más aún, es probable que la mayoría de los estudiantes de medicina privilegie aquel o aquellos estilos que les han permitido ser exitosos en su formación académica y que no estén dispuestos a cambiarlos en forma espontánea. El ideal sería que los aprendedores desarrollaran en forma equilibrada todas sus capacidades para conseguir un aprendizaje significativo, ya que las situaciones a las que se enfrentarán en su formación futura requerirán de diferentes estrategias de aprendizaje [3]. Por lo tanto, sería un deber de las Facultades de Medicina buscar alternativas metodológicas para potenciar el desarrollo de los estilos activos y pragmáticos, en la misma medida que los estilos reflexivos y teóricos. El estudiante más capacitado será aquel capaz de aprender en cualquier situación, ya que aprovechará mejor las oportunidades de aprendizaje que se le presenten a lo largo de su vida. Afortunadamente, las preferencias por determinados estilos de aprendizaje no son inmutables. Según Alonso [3], ellos evolucionan de acuerdo con nuestra edad cronológica y nuestras experiencias. Podemos mejorar cada uno de los estilos a través de la metacognición y prácticas docentes adecuadas que permitan reforzar nuestros estilos preferentes y potenciar nuestros estilos menos desarrollados [1,9]. Los profesores deben ser capaces de utilizar distintos estilos de enseñanza para facilitar el aprendizaje de alumnos con distintos estilos de aprendizaje y fomentar la flexibilidad de los alumnos en el uso de los diferentes estilos.

En la relación entre estilo preferente de aprendizaje y contexto geográfico es donde se aprecian las diferencias más marcadas en nuestro estudio. Mientras que en las Universidades de Chile, del País Vasco, y Nacional de Cuyo se mantienen los estilos reflexivo y teórico como los predominantes, en las de Zaragoza y Sucre se agrega a ellos el estilo pragmático. Llama la atención que en ninguna de las universidades que participaron en este estudio se haya encontrado preferencia por el estilo de aprendizaje activo, el cual se basa en la experiencia directa y debería desarrollarse 


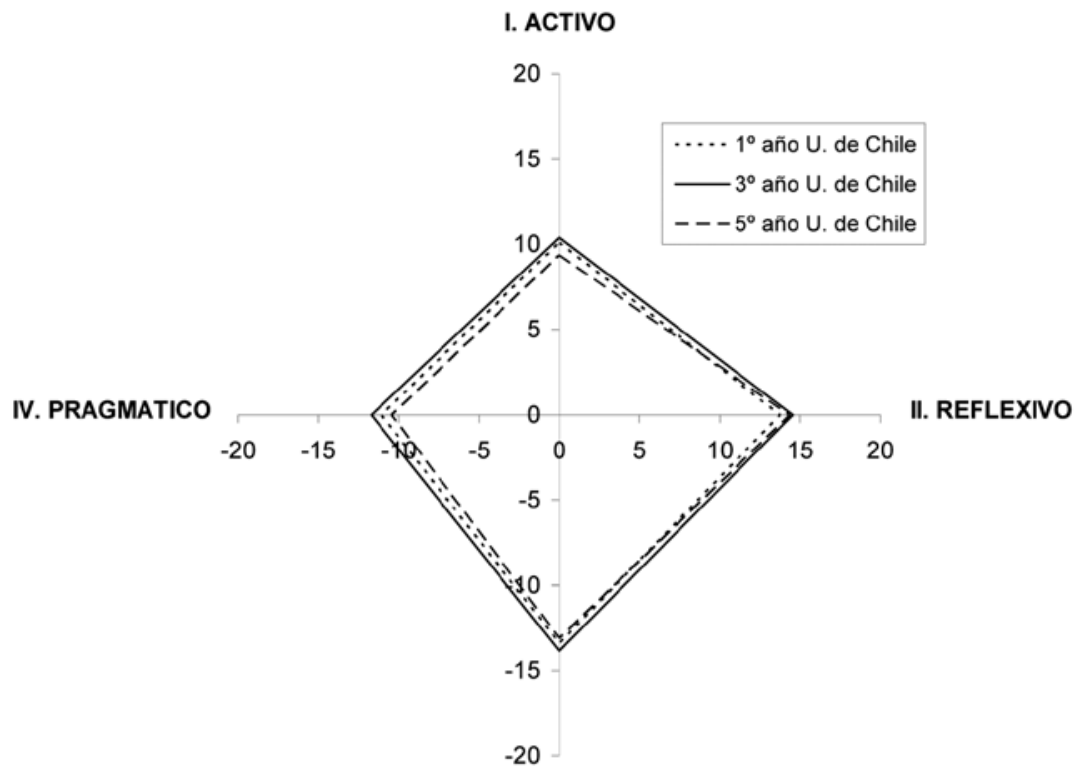

III. TEORICO

\begin{tabular}{|c|c|c|c|c|}
\hline Curso & Activo & Reflexivo & Teórico & Pragmático \\
\hline $1^{\circ}$ & $10,1 \pm 0,4$ & $13,7 \pm 0,4 *$ & $13,4 \pm 0,3 *$ & $11,1 \pm 0,4$ \\
\hline $3^{\circ}$ & $10,4 \pm 0,5$ & $14,5 \pm 0,4 *$ & $13,9 \pm 0,4^{*}$ & $11,7 \pm 0,4$ \\
\hline $5^{\circ}$ & $10,0 \pm 0,5$ & $14,9 \pm 0,4 *$ & $13,1 \pm 0,4 *$ & $10,4 \pm 0,5$ \\
\hline
\end{tabular}

Figura 6. Puntuaciones promedio obtenidas para cada estilo de aprendizaje en la muestra de estudiantes de la Facultad de Medicina de la Universidad de Chile (Chile). ${ }^{*} p<0,05$ comparando entre los estilo de aprendizaje de cada curso. No se aprecian diferencias significativas entre los tres cursos para cada estilo de aprendizaje.

procurando que los estudiantes, por ejemplo, se enfrenten a situaciones nuevas que los obliguen a resolver problemas, trabajar en equipo, introducir cambios que eviten la rutina, hacer presentaciones, discutir, dramatizar...

La relación del contexto geográfico con los estilos de aprendizaje según Kolb, evidenció que los estudiantes de las dos universidades españolas son preferentemente asimiladores, característica que ya había sido señalada por otros autores $[1,5]$, estos estudiantes utilizan la conceptualización abstracta y la observación reflexiva, siendo capaces de percibir gran cantidad de información y procesarla en forma lógica, se interesan muy poco por las personas y por lo social. En cambio, los estudiantes chilenos de primer y tercer curso, mostraron ser marcadamente acomodadores, es decir su punto fuerte es hacer cosas, llevar a cabo proyectos y experimentos e involucrarse en situaciones nuevas. Esta misma preferencia se observó en los estudiantes de tercer año de la Universidad Nacional de Cuyo. Los estudiantes bolivianos se distribuyeron entre los estilos asimiladores y convergentes, es decir se interesan por los conceptos abstractos pero requieren llevar a la práctica las teorías aprendidas. Todas estas diferencias resultan difíciles de explicar, ya que el diferente contexto geográfico necesariamente conlleva otras variables socioculturales que, por la naturaleza de este estudio, no fueron evaluadas. 
En conclusión, en este trabajo las principales diferencias entre los estilos de aprendizaje de los estudiantes de Medicina se relacionan con el contexto geográfico, más que con los diferentes currículos, o a lo largo de los distintos cursos de la carrera. Las cinco facultades que participaron en el estudio presentaron sesgos en las preferencias de los estudiantes por los distintos estilos de aprendizaje. Una recomendación sería diseñar diferentes estrategias para equilibrar dichos sesgos, incorporando actividades que desarrollen en particular el aprendizaje activo en todas ellas y el aprendizaje pragmático en las Universidades de Chile, Nacional de Cuyo y del País Vasco. La aplicación de cuestionarios, como el CHAEA, permite no solo identificar el estilo de aprendizaje preferente de una población, sino que también proporciona una valiosa información para que los docentes adecuen su metodología y planifiquen diversas estrategias de formación a futuro, de manera de potenciar el desarrollo de las capacidades individuales para un aprendizaje significativo en todos los estudiantes, sin olvidar la utilidad que puede tener en ellos el desarrollo del autoaprendizaje y la metacognición, al aumentar el conocimiento acerca de sus fortalezas y debilidades como aprendedores.

\section{Bibliografía}

1. Escanero JF, González-Haro C, Ezquerra L, Borque L. Diseño e implementación de una práctica de metacognición en la asignatura de Fisiología Humana (Facultad de Medicina). Educ Med 2009; 12: 47-53.
2. Monereo C, Pozo JI. La cultura educativa en la universidad: nuevos retos para profesores y alumnos. In Monereo C, Pozo JI, eds. La universidad ante la nueva cultura educativa. Enseñar y aprender para la autonomía. Madrid: Síntesis; 2003. p. 15-30.

3. Alonso C, Gallego D, Honey P. Los estilos de aprendizaje. Procedimientos de diagnóstico y mejora. Bilbao: Mensajero; 1994.

4. Curry L. Cognitive and learning styles in medical education. Acad Med 1999; 74: 409-13.

5. Kolb DA. The Learning Style Inventory: technical manual. Boston: McBer; 1976.

6. Kolb DA. Learning Style Inventory, version 3. Technical specifications. Boston: TRG Hay/McBer; 1999.

7. Honey P, Munford A. The manual of learning styles. Maidenhead, Berkshire: P. Honey, Ardingly House; 1986.

8. Kolb DA. Experiential learning: experience as the source of learning and development. Englewood Cliffs, NJ: Prentice Hall; 1984.

9. Kolb AY, Kolb DA. Learning styles and learning spaces: enhancing experiential learning in higher education. Acad Manag Learn Educ 2005; 4: 193-212.

10. Correa JE. Identificación de los estilos de aprendizaje en los estudiantes de fisiología del ejercicio en la Facultad de Rehabilitación y Desarrollo Humano. Rev Cienc Salud Bogotá 2006; 4: 41-53.

11. Soria M, Guerra M, Lou M, Pié J, Escanero JF. Estilos de aprendizaje de los estudiantes de ciencias de la salud. Educ Med 2005; 8: 145.

12. Ordoñez FJ, Rosety-Rodríguez M, Rosety-Plaza M. Análisis de los estilos de aprendizaje predominantes en los estudiantes de Ciencias de la Salud. Enfermería Global (Servicio de Publicaciones de la Universidad de Murcia); 2003; 3. URL: www.um.es/eglobal/3/03c04.html.

13. Canalejas M, Pérez M, Martín L, Pineda M, Vera M, Soto $\mathrm{M}$, et al. Learning styles in nursing students. Educ Med 2005; 8: 83-90. 